\title{
A GENERALIZATION OF WEIERSTRASS' AND FEKETE'S MEAN-VALUE THEOREMS*
}

\section{BY MORRIS MARDEN}

1. Introduction. Three of our recent papers have dealt with the problem of determining zero-free regions for certain sums of rational functions. $\dagger$ Our study was based largely upon the familiar principle that a vector sum $\sum \overrightarrow{O P}_{j}$ cannot vanish if all the points $P_{j}$ lie in the same angle with vertex at $O$ and with a magnitude of less than $\pi$ radians. In the present paper, we propose to employ the same principle in order to generalize two mean-value theorems.

The first of these theorems will be that of Weierstrass. $\ddagger$ If $g(z)$ is real and positive on a curve $C: z=\psi(t),(a \leqq t \leqq b)$, and if $w=f(z)$ maps $C$ one-to-one continuously on a regular curve $\Gamma$ of the w-plane, any convex region containing $\Gamma$ also contains the point $\sigma$ as defined by the equation

$$
\int_{a}^{b} f(t) g(t) d t=\sigma \int_{a}^{b} g(t) d t .
$$

In $\S 2$, the above hypothesis on $g(z)$ will be replaced by the more extensive one that $g(z)$ assume any value in a given angular domain with an opening of less than $\pi$ radians. The point $\sigma$ will then be free to lie in a region which includes at least the convex region of Weierstrass' theorem. This larger region will be determined in $\$ 2$ and shown to be a "best approximation" to the position of $\sigma$.

The other theorem to be considered is one due to Fekete. $\S$ If a polynomial $P(z)$ of degree $n$ takes on at $z=k_{1}$ and $z=k_{2}$ the unequal values $r_{1}$ and $r_{2}$, then it takes on every value of the line-

* Presented to the Society, December 30, 1931.

$\dagger$ This Bulletin, vol. 35 (1929), pp. 363-370, and Transactions of this Society, vol. 32 (1930), pp. 658-668, and vol. 33 (1931), pp. 934-944.

¥ See Osgood, Lehrbuch der Funktionentheorie, 1923, vol. I, p. 212.

$\S$ M. Fekete, Acta Universitatis Hungaricae, vol. 1 (1923), pp. 98-100. Also Pólya-Szegö, Aufgaben und Lehrsätze, vol. I, p. 257. 
segment $r_{1} r_{2}$ at least once within or on a circle with center at $z$ $=\left(k_{1}+k_{2}\right) / 2$ and with a radius $\frac{1}{2}\left|k_{1}-k_{2}\right| \operatorname{ctn}(\pi /(2 n))$.

This theorem has been extended by J. v. Sz. Nagy* as follows: If $P(z)$ is a polynomial of degree $n$, if $P\left(k_{j}\right)=r_{j},(j=1$, $2, \cdots, m)$, and if $K$ and $R$ are the smallest convex polygons enclosing the points $k_{j}$ and $r_{j}$, respectively, then $P(z)$ takes on any value $\sigma$ in $R$ at least once within the smallest circle from whose circumference $K$ subtends an angle $\phi \leqq \pi / n$.

An equivalent way of expressing Nagy's theorem is that if $P(z)$ is a polynomial of degree $n$, if $k_{1}, k_{2}, \cdots, k_{m}$ are points of a convex region $K$, and if $\alpha_{1}, \alpha_{2}, \cdots, \alpha_{m}$ are positive real numbers, then $P(z)$ assumes the value $\sigma$, where

$$
\sigma \sum_{j=1}^{m} \alpha_{j}=\sum_{j=1}^{m} \alpha_{j} r_{j},
$$

at least once in the smallest circle from whose circumference $K$ subtends an angle $\phi \leqq \pi / n$.

In $\S 3$, the above hypothesis on the $\alpha_{i}$ will be replaced by the less restrictive one that the $\alpha_{j}$ all lie in an angular domain with an opening not exceeding $\pi$. The region in which $P(z)$ will at least once assume the value $\sigma$ will necessarily be larger than that required under Fekete's or Nagy's assumptions. This larger region will be described in $\$ 3$. Finally, $\$ 4$ will be devoted to further discussion of Fekete's work.

2. Weierstrass' Theorem. We may state our generalization of Weierstrass' theorem as follows.

THEOREM 1. Given $C: z=\psi(t),(a \leqq t \leqq b)$, a rectifiable curve in the z-plane, $F$ a convex region in the w-plane and $G$ a region in the $w$-plane composed of the points lying in or on an angle with vertex at the origin and with a magnitude of $\gamma<\pi$. Let $\Sigma$ be the starshaped region $\dagger$ consisting of all points wat which $F$ subtends an angle of not less than $\pi-\gamma$.

If $f(z)$ and $g(z)$ are any two functions which on $C$ are continuous except for a finite number of finite jumps and which on $C$ assume only values within $F$ and $G$ respectively, then the point $\sigma$ as defined by the equation

* Jahresbericht der Vereinigung, vol. 32 (1923), pp. 307-309.

$\dagger$ See M. Marden, Transactions of this Society, vol. 32 (1930), pp. 658-9. 


$$
\int_{a}^{b} f(t) g(t) d t=\sigma \int_{a}^{b} g(t) d t
$$

lies in $\Sigma$. Conversely, if $\sigma$ is any point of $\Sigma$, functions $f(z)$ and $g(z)$ fulfilling the above conditions can be found so that equation (1) is satisfied.

In demonstrating this theorem, we may suppose that $G$ is defined by the inequality

$$
0 \leqq \arg w \leqq \gamma .
$$

No loss of generality ensues since multiplication of equation (1) by $e^{i \theta}$ does not affect the value of $\sigma$.

To prove the first part of the theorem, let us assume that $\sigma$ lies outside of $\Sigma$. This means that for all points $z$ on $C$, the vector $f(z)-\sigma$ lies within an angle of magnitude less than $\pi-\gamma$. That is to say, there exists a positive real number $\delta$ such that for all $z$ on $C$

$$
\begin{aligned}
& 0 \leqq \arg [f(z)-\sigma]-\delta<\pi-\gamma \\
& 0 \leqq \arg g(z)[f(z)-\sigma]-\delta<\pi
\end{aligned}
$$

Hence

$$
\int_{a}^{b} g(t)[f(t)-\sigma] d t \neq 0,
$$

in contradiction to the fact that $\sigma$ satisfies equation (1). Consequently, the point $\sigma$ must lie in $\Sigma$.

To prove the second part of the theorem, let us suppose $\sigma$ to be any point of $\Sigma$. Then $\sigma$ does or does not also lie in $F$. If $\sigma$ lies in $F$, we need only choose $f(z)=\sigma$ and $g(z)=1$. For then we shall have

$$
\int_{a}^{b} g(t)[f(t)-\sigma] d t=(b-a)(\sigma-\sigma)=0 .
$$

If $\sigma$ does not lie in $F$, the angle subtended at $\sigma$ by $F$ will be $\pi-\gamma^{\prime}$, where $0 \leqq \gamma^{\prime} \leqq \gamma$. That is to say, there exist in $F$ two points $\alpha$ and $\beta$, such that

$$
\arg \frac{\beta-\sigma}{\alpha-\sigma}-\pi-\gamma^{\prime} .
$$


Now we have but to make

$$
\begin{array}{ll}
g(z)=\frac{|\beta-\sigma|}{c-a}, f(z)=\alpha, & (a \leqq t \leqq c), \\
g(z)=\frac{|\alpha-\sigma|}{b-c} e^{i \gamma^{\prime}}, \quad f(z)=\beta, & (c \leqq t \leqq b),
\end{array}
$$

for then

$$
\int_{a}^{b} g(t)[f(t)-\sigma] d t=|\beta-\sigma|(\alpha-\sigma)+|\alpha-\sigma|(\beta-\sigma) e^{i \cdot \gamma^{\prime}} .
$$

The right-hand side of this expression is zero since

$$
\frac{|\beta-\sigma|}{|\alpha-\sigma|} e^{-i \gamma^{\prime}}=-\frac{\beta-\sigma}{\alpha-\sigma} \text {. }
$$

In Theorem 1, thus proved, it is to be noted that, when $\gamma$ approaches zero, region $\Sigma$ approaches $F$; that is, our theorem reduces essentially to Weierstrass'; and that, when $\gamma$ approaches $\pi$, region $\Sigma$ expands indefinitely; that is, the magnitude of the angular domain $G$ cannot be enlarged beyond $\pi$ without vitally changing the theorem.

Two corollaries may be deduced at once from Theorem 1.

Corollary 1. If $\zeta(z)$ and $F(z)$ are continuous (except for a finite number of finite jumps) on a curve $C: z=\psi(t),(a \leqq t \leqq b)$, and if on $C$

$$
0 \leqq \arg \zeta(z) \leqq \gamma<\pi,
$$

there exists a number $\sigma,|\sigma| \leqq \sec (\gamma / 2)$, such that

$$
\int_{t=a}^{t=b} \zeta(z) F(z) d z=\sigma \int_{t=a}^{t=b} \zeta(z)|F(z)| d s .
$$

For let us set in Theorem 1

$$
f(z)=e^{i \arg F(z)} \frac{d z}{d s}, \quad g(z)=\zeta(z)|F(z)| .
$$

Since $|f(z)|=1$, it follows that $|\sigma| \leqq \sec (\gamma / 2)$.

Corollary 2. Under the same conditions as in Corollary 1, if $|F(z)| \leqq M$ on $C$, a number $\sigma,|\sigma| \leqq M \sec (\gamma / 2)$, exists such that 


$$
\int_{t=a}^{t=b} \zeta(z) F(z) d z=\sigma \int_{t=a}^{t=b} \zeta(z) d s .
$$

For, let us choose

$$
f(z)=F(z) \frac{d z}{d s} \text { and } \quad g(z)=\zeta(z) .
$$

Then, since $|f(z)| \leqq M$, we have $|\sigma| \leqq M \sec (\gamma / 2)$.

These two corollaries lead to the following inequalities:

$$
\begin{aligned}
& \left|\int_{a}^{b} \zeta(z) F(z) d z\right| \leqq \sec \frac{\gamma}{2}\left|\int_{a}^{b} \zeta(z) F(z) d s\right|, \\
& \left|\int_{a}^{b} \zeta(z) F(z) d z\right| \leqq M \sec \frac{\gamma}{2}\left|\int_{a}^{b} \zeta(z) d s\right| .
\end{aligned}
$$

The last inequality is an extension of the Darboux* theorem that, if $\zeta(z)$ is real and positive on $C$ and $\xi$ a suitable point on $C$,

$$
\left|\int_{a}^{b} \zeta(t) F(t) d t\right| \leqq|F(\xi)| \int_{a}^{b} \zeta(t) d t .
$$

3. Fekete's Theorem. Our generalization of Fekete's Theorem and of its extension by Nagy may be put in the following terms.

TheOREM 2A. Given the positive integer $n$, the convex region $K$ and the angular domain $A$ with vertex at the origin and with a magnitude of $\gamma<\pi$. Let $S$ be the star-shaped region consisting of all points at which $K$ subtends an angle of not less than $(\pi-\gamma) / n$.

If $P(z)$ is any polynomial of degree $n$ and if $k_{j}$ and $\alpha_{j}(j=1$, $2, \cdots, m)$ are any points of $K$ and $A$ respectively, then $P(z)$ assumes the value $\sigma$, where

$$
\sigma \sum_{j=1}^{m} \alpha_{j}=\sum_{j=1}^{m} \alpha_{j} P\left(k_{j}\right),
$$

at least once in $S$. Conversely, if $s$ is any point of $S$, there exists a polynomial $P(z)$ of degree $n$ which for a suitable choice of points $k_{j}$ and $\alpha_{j}$ in $K$ and $A$ will satisfy the relation

$$
P(s) \sum_{j=1}^{m} \alpha_{j}=\sum_{j=1}^{m} \alpha_{j} P\left(k_{j}\right) .
$$

\footnotetext{
* Osgood, Lehrbuch der Funktionentheorie, p. 213.
} 
In the proof of this theorem we may suppose without loss of generality that region $A$ is defined by the inequality

$$
0 \leqq \arg z \leqq \gamma<\pi
$$

Let us write

$$
P(z)-\sigma=C\left(z-z_{1}\right)\left(z-z_{2}\right) \cdots\left(z-z_{n}\right) .
$$

If all the $z_{j}$ were outside of $S$, the rays from any one $z_{j}$ to all the $k_{i}$ would lie within an angle of less than $(\pi-\gamma) / n$. That is to say, a constant $\delta_{j}$ would exist such that for all $i$

$$
\begin{aligned}
& 0 \leqq \arg \left(k_{i}-z_{j}\right)-\delta_{j}<\frac{\pi-\gamma}{n}, \\
& 0 \leqq \arg \left[P\left(k_{i}\right)-\sigma\right]-\arg C-\sum_{j=1}^{m} \delta_{j}<\pi-\gamma, \\
& 0 \leqq \arg \alpha_{i}\left[P\left(k_{i}\right)-\sigma\right]-\arg C-\sum_{j=1}^{m} \delta_{j}<\pi,
\end{aligned}
$$

and hence

$$
\sum_{i=1}^{m} \alpha_{i}\left[P\left(k_{i}\right)-\sigma\right] \neq 0
$$

in contradiction to the hypothesis that (2) is satisfied. Consequently, $P(z)$ assumes the value $\sigma$ at least once in $S$.

To prove the converse proposition, let us choose any point $s$ in $S$. Point $s$ is or is not also in $K$. If it is in $K$, we may choose $m=1, \alpha_{1}=1$, and $k_{1}=s$, thus satisfying relation (3). If it is not in $K$, the angle subtended by $K$ at $s$ has a value $\left(\pi-\gamma^{\prime}\right) / n$, where $0<\gamma^{\prime} \leqq \gamma$. That is to say, there exist in $K$ two points $k_{1}$ and $k_{2}$ such that

$$
\arg \frac{k_{2}-s}{k_{1}-s}=\frac{\pi-\gamma^{\prime}}{n} .
$$

Let us now set $m=2$,

$$
\alpha_{1}=\left|k_{2}-s\right|^{n}, \quad \alpha_{2}=\left|k_{1}-s\right|^{n} e^{i \gamma^{\prime}},
$$

and

$$
P(z)=P(s)+(z-s)^{n} .
$$


Then

$$
\begin{aligned}
\alpha_{1}\left[P\left(k_{1}\right)-P(s)\right] & +\alpha_{2}\left[P\left(k_{2}\right)-P(s)\right] \\
= & \left|k_{2}-s\right|^{n}\left(k_{1}-s\right)^{n}+\left|k_{1}-s\right|^{n}\left(k_{2}-s\right)^{n} e^{i \gamma^{\prime}} .
\end{aligned}
$$

By using the definition of $\gamma^{\prime}$, it is obvious that the right-hand side is zero.

It is to be noticed first that, if $K$ is specialized to be a circle whose radius is $r, \Sigma$ becomes a concentric circle $S$ whose radius is $r \csc [(\pi-\gamma) /(2 n)]$.

Secondly, when $\gamma=0$, our theorem provides an approximation to one root of $P(z)-\sigma=0$ which in general is better than Nagy's, coinciding with his only when $K$ is a circle.

Finally, inasmuch as the results in Theorem $2 \mathrm{~A}$ do not depend upon $m$, we may state the following theorem.

THEOREM 2B. Let $P(z)$ be an arbitrary polynomial of degree $n$ and $C: z=\psi(t),(a \leqq t \leqq b)$, a rectifiable curve drawn within a given convex region $K$. On $C$ let $\alpha(z)$ be continuous and assume only values within an angular domain whose vertex is at the origin and whose magnitude is $\gamma<\pi$. Then the star-shaped region $S$ consisting of all points at which $K$ subtends an angle of not less than $(\pi-\gamma) / n$ contains at least one point s such that

$$
\int_{a}^{b} P(t) \alpha(t) d t=P(s) \int_{a}^{b} \alpha(t) d t .
$$

4. Addenda. As Professor Fekete has kindly pointed out to me, he has already proved Theorem 2A (an analog to Bolzano's theorem) for $m=2 .^{*}$

Since $\int_{a}^{b} \alpha(z) d t \neq 0$ in Theorem 2B, it follows that, if $\int_{a}^{b} P(z) \alpha(z) d t=0, P(z)$ vanishes at least once in $S$. For $\gamma=0$, this result coincides with one due to Fekete. ${ }^{*}$ For $\alpha(z) \equiv 1, \psi(t) \equiv t$ and $P(z)=Q^{\prime}(z)$ where $Q(z)$ is any polynomial of degree $n$, it yields an analog to Rolle's theorem also due to Fekete. $\dagger$

Through a chain of arguments similar to those employed in proving Theorems $2 \mathrm{~A}$ and $2 \mathrm{~B}$, we may establish the following more general thèorem.

* Mathematische Zeitschrift, vol. 22 (1925), p. 2, and Jahresbericht der Vereinigung, vol. 34 (1926), p. 221.

$\dagger$ Mathematische Zeitschrift, vol. 22 (1925), p. 4. 
THEOREM 3. Given two positive integers $p$ and $q$, a convex region $K$ and an angular domain $A$ with vertex at the origin and with $a$ magnitude of $\gamma<\pi$. Let $S$ be the star-shaped region consisting of all the points from which $K$ subtends an angle of not less than $(\pi-\gamma) /(m+q)$, where $m=\max (p, q)$. Let $C: z=\psi(t),(a \leqq t \leqq b)$, be a rectifiable curve drawn in $K$, and let $\alpha(z)$ be a function which is continuous on $C$ and which assumes on $C$ only values in $A$. Finally, let $P(z)$ and $Q(z)$ be any two polynomials of degrees $p$ and $q$ respectively such that $R(z)=P(z) / Q(z)$ is irreducible and has no poles in $S$. Then in $S$ there exists at least one point s such that

$$
\int_{a} R(z) \alpha(z) d t=R(s) \int_{a}^{b} \alpha(z) d t .
$$

Theorem 3 reduces essentially to one due to Fekete* for

$$
\psi(t) \equiv k_{1}, a \leqq t \leqq \frac{1}{2}(a+b) ; \psi(t) \equiv k_{2}, \quad \frac{1}{2}(a+b)<t \leqq b .
$$

University of Wisconsin, Milwaukee

* The above Acta article, p. 236. 